\title{
Qian Yang Yu Yin Granule-containing serum inhibits angiotensin II-induced proliferation, reactive oxygen species production, and inflammation in human mesangial cells via an NADPH oxidase 4-dependent pathway
}

Kang Ding ${ }^{1,2+}$, Yan Wang ${ }^{4 \dagger}$, Weimin Jiang ${ }^{3}$, Yu Zhang ${ }^{5}$, Hongping Yin ${ }^{4^{*}}$ and Zhuyuan Fang ${ }^{3^{*}}$

\begin{abstract}
Background: Qian Yang Yu Yin Granule (QYYYG), a traditional Chinese herbal medicine, has been indicated for renal damage in hypertension for decades in China, but little remains known regarding its underlying molecular mechanism. Therefore, we performed the current study in order to investigate the underlying molecular mechanism of QYYYG in the treatment of hypertensive renal damage.

Methods: We hypothesize that QYYYG relieves hypertensive renal injury through an angiotensin II (Ang II)-nicotinamide adenine dinucleotide phosphate (NAPDH)-oxidase (NOX)-reactive oxygen species (ROS) pathway. In this study, we investigated the effects of QYYYG-containing serum (QYGS) in human mesangial cells (HMCs) against Ang II-induced cell proliferation, ROS production, and inflammation through the seropharmacological method.

Results: We found that QYGS could inhibit cell proliferation in Ang II-treated HMCs. In addition, QYGS considerably suppressed production of ROS, decreased mRNA and protein expression of NAPDH-oxidase 4 (NOX4), p22 $2^{\text {phox }}$, and activated Ras-related C3 botulinum toxin substrate 1 (GTP-Rac1); as well as counteracted the up-regulation of inflammatory markers including tumor necrosis factor-a (TNF-a), nuclear factor-KB (NF-KB) p65, and interleukin 6 (IL-6). These effects were further confirmed in HMCs transfected with specific small interfering RNA (siRNA) targeting NOX4.

Conclusions: Taken together, these results suggest that a NOX4-dependent pathway plays an important role in regulating the inhibitory effect of QYGS. Our findings provide new insights into the molecular mechanisms of QYYYG and their role in the treatment of hypertensive nephropathy.
\end{abstract}

Keywords: Angiotensin II, NADPH oxidase, Reactive oxygen species, Human mesangial cells, Qian Yang Yu Yin Granule

\footnotetext{
*Correspondence: yinhongping@cpu.edu.cn; zhuyuan_fang@163.com

${ }^{\dagger}$ Equal contributors

${ }^{4}$ School of Life Science and Technology, China Pharmaceutical University,

Nanjing 210009, China

3 Jiangsu Province Hospital of Traditional Chinese Medicine, No.155

Hanzhong Road, Nanjing 210029, China

Full list of author information is available at the end of the article
} 


\section{Background}

Hypertension is one of the most common risk factors for cardiovascular and renal diseases [1]. Recently, it has been widely accepted that the renin-angiotensin system (RAS) plays an essential role in the pathophysiology of hypertension-related renal damage [2]. Numerous studies have demonstrated that angiotensin II (Ang II), the central effector molecule of the RAS, significantly contributes to the induction of irreversible renal damage through the direct or indirect stimulation of glomerular mesangial expansion, glomerular basement membrane reduplication, and extracellular matrix (ECM) deposition [3]. In addition, researchers have found that Ang II is able to induce contraction of glomerular mesangial cells, which contributes to the development of glomerular atrophy and sclerosis, resulting in progressive renal dysfunction [4,5]. Therefore, fully understanding the underlying molecular mechanism of Ang II-induced glomerular pathological changes has important implications for the prevention and treatment of renal damage in hypertension.

In recent years, a considerable number of studies have identified several specific signaling pathways that mediate the pathogenic actions of Ang II, of which a nicotinamide adenine dinucleotide phosphate (NADPH)oxidase (NOX)-dependent pathway plays a pivotal role [6] Previous investigations have confirmed that Ang II induces reactive oxygen species (ROS) generation through the NOX system [5,7]. Then, oxidative stress from the ROS stimulates ROS-sensitive inflammatory pathways, such as nuclear factor- $k \mathrm{~B}(\mathrm{NF}-\mathrm{kB})$ cascade, to produce a number of pro-inflammatory mediators and cytokines $[8,9]$. These pro-inflammatory factors, together with Ang II-induced oxidative stress, are able to cause renal vascular remodeling and injury to the endothelia cells of renal small arteries. These effects decrease renal blood supply, ultimately leading to ischemic renal parenchymal injury [10]. Hence, the blockade of Ang II has been considered a major therapeutic strategy for hypertensive nephropathy.

Angiotensin receptor blockers (ARBs), the most widelyused anti-hypertensive drugs, have been recommended for use in the management of hypertensive nephropathy by the 2013 European guidelines for the management of arterial hypertension [11]. Numerous recent studies also have demonstrated the protective effects of ARBs against Ang II-induced glomerular pathological changes through blocking oxidative stress and oxidative stress-mediated inflammatory responses [12-14]. However, due to the pathogenic complexity of hypertensive nephropathy, there is still an increasing demand for novel and effective therapeutics.

In recent years, traditional Chinese medicine has shown its advantage against hypertension and hypertensionrelated complications [15]. Qian Yang Yu Yin Granule
(QYYYG), a traditional Chinese herbal medicine, has been indicated for renal damage in hypertension for decades in China, but little remains known regarding its underlying molecular mechanism. Polygoni Multiflori Radix, one of the major ingredients of QYYYG, has been found to induce potent antioxidant and anti-inflammatory effects $[16,17]$. Therefore, we hypothesize that QYYYG relieves hypertensive renal injury through an Ang II-NOX-ROS pathway. In the present study, we investigated the effects of QYYYG-containing serum (QYGS) in human mesangial cells (HMCs) against Ang II-induced cell proliferation, ROS production, and inflammation through the seropharmacological method. We found that these effects of QYGS are likely to be mediated through a NOX4-dependent pathway, which may possibly be a molecular mechanism behind the pharmacological effects of QYYYG.

\section{Methods}

\section{Reagents}

QYYYG (batch No.1307004) was provided by the Institute of Chinese Traditional Medicine of Jiangsu Province (Nanjing, China). Detailed information on the components of QYYYG and their amounts is listed in Table 1. The preparation process of QYYYG was as follows: All components were mixed in proportion. The mixture was decocted with 10-fold amount of water (volume/weight) twice, for $1 \mathrm{~h}$ each time. The resultant filtrates were combined and concentrated to a relative density of $1.2 \mathrm{~g} / \mathrm{ml}$ (at $60^{\circ} \mathrm{C}$ ). After adding stevioside to a concentration of $1 \%$ (weight/volume), the concentrated filtrate was mixed with dextrin at a weight ratio of 1:1 and granulated in a fluidized bed granulator. The resulting granules were dried at $60^{\circ} \mathrm{C}$ for $24 \mathrm{~h}$ and then stored at $4^{\circ} \mathrm{C}$ for future use.

According to the Pharmacopoeia of the People's Republic of China (2010 edition) and the Quality Specifications of Chinese Traditional Medicine of Jiangsu Province, thin-layer chromatography (TLC) and high performance liquid chromatogram (HPLC) methods were employed to control the quality of QYYYG (Table 2). Valsartan (Diovan ${ }^{\circ}$, batch No. X1584) was provided by Norvatis (Basel, Switzerland).

Ang II was purchased from Sigma (St. Louis, MO, USA). Dulbecco's modified Eagle's medium (DMEM), trypsin, and fetal bovine serum (FBS) were from Life Technologies (Carlsbad, CA, USA). Dimethyl sulfoxide (DMSO) and 3-(4,5-dimethyl-2-thiazolyl)-2,5-diphenyl tetrazolium bromide (MTT) were purchased from Amresco (Solon, OH, USA). TRNzol ${ }^{\circ}$ Plus reagent was from Biouniquer (Nanjing, China).

\section{Cell culture}

HMCs were provided by the Institute of Life Science and Technology, China Pharmaceutical University (Nanjing, China). The cells were cultured in DMEM 
Table 1 The components of QYYYG

\begin{tabular}{llll}
\hline Components & Chinese name & Origin & Amount used (g) \\
\hline Polygoni Multiflori Radix & He Shou Wu & Polygonum multiflorum Thunb. & 180 \\
Herba Bidentis Bipinnatae & Gui Zhen Cao & Bidens pilosa Linnaeus & 110 \\
Corni Fructus & Shan Zhu Yu & Cornus officinalis & 108 \\
Scrophulariae Radix & Xuan Shen & Scrophularia ningpoensis & 180 \\
Alismatis Rhizoma & Ze Xie & Alisma orientalis & 180 \\
Cyathulae Radix & Chuan Niu Xi & Cyathula officinalis & 170 \\
\hline
\end{tabular}

supplemented with 10\% FBS, $2 \mathrm{mM}$ glutamine, $100 \mathrm{U} / \mathrm{ml}$ penicillin, and $100 \mathrm{mg} / \mathrm{ml}$ streptomycin at $37^{\circ} \mathrm{C}$ in a $5 \%$ $\mathrm{CO}_{2}$ atmosphere.

\section{Preparation of QYYYG-containing serum}

Thirty Wistar rats (age of 14 weeks, weighing between 260 and 320 g) were provided by the Experimental Animal Center of Yangzhou (Yangzhou, China). The animal experiments were conducted in accordance with the guidelines as set forth by the National Research Council. The study protocol was approved by the Animal Ethics Committee of Nanjing University of Traditional Chinese Medicine.

All animals were maintained under temperaturecontrolled $\left(22 \pm 2^{\circ} \mathrm{C}\right)$ and humidity-controlled $(55 \pm 5 \%)$ conditions in 12-hour light/dark cycles with free access to sterile normal food and water. After an acclimation period of one week, the animals were randomly divided into three groups: the QYGS group, the valsartancontaining serum (VS) group, and the control serum group ( $n=10$ animals per group). Animals in the QYGS group and VS group were orally administered $2.5 \mathrm{~g} / \mathrm{kg}$ of QYYYG (10-fold clinic dosage) and $13.3 \mathrm{mg} / \mathrm{kg}$ of valsartan (10-fold of clinic dosage) once daily for 7 days. Both drugs were suspended in $3 \mathrm{ml}$ of distilled water. One hour after the last administration of QYYYG or valsartan on the seventh day, whole blood was obtained from the retro-orbital venous plexus of the animals in all groups. For all groups, serum was isolated by centrifugation at $3000 \mathrm{rpm}$ for $10 \mathrm{~min}$ after standing at room temperature (RT) for $30 \mathrm{~min}$, and then mixed with serum

Table 2 Quality evaluation of QYYYG

\begin{tabular}{lll}
\hline Major constituents & $\begin{array}{l}\text { Method of } \\
\text { determination }\end{array}$ & $\begin{array}{l}\text { Quality } \\
\text { specifications }\end{array}$ \\
\hline $\begin{array}{l}\text { 2,3,5,4'-tetrahydroxystilbene- } \\
\text { 2-O- } \beta \text {-D-glucopyranoside }\end{array}$ & HPLC & $\begin{array}{l}>6 \mathrm{mg} \text { per } 10 \mathrm{~g} \\
\text { QYYYG }\end{array}$ \\
Polygoni Multiflori Radix & TLC & Contained \\
Herba Bidentis Bipinnatae & TLC & Contained \\
Corni Fructus & TLC & Contained \\
Cyathulae Radix & TLC & Contained
\end{tabular}

HPLC: high performance liquid chromatogram, TLC: thin-layer chromatography. from the same group. Then, the serum was inactivated in a $56^{\circ} \mathrm{C}$ environment for $30 \mathrm{~min}$ and filtered with $0.22 \mu \mathrm{m}$ pore-size membrane. The filtered serum was aliquoted and stored at $-20^{\circ} \mathrm{C}$ for future use.

\section{Cell proliferation assay}

MTT assay was used to evaluate the proliferation ability of Ang II-treated HMCs. Cells were transferred onto 96-well plates containing DMEM supplemented with $10 \%$ FBS. The optimal cell number was found to be $1 \times 10^{4}$ cells/well. Cells were then treated with varying concentrations of Ang II $\left(0,10^{-3}, 10^{-4}, 10^{-5}, 10^{-6}\right.$, and $\left.10^{-7} \mathrm{mmol} / \mathrm{L}\right)$. After $24 \mathrm{~h}, 20 \mu \mathrm{l} \mathrm{MTT}(5 \mathrm{mg} / \mathrm{ml})$ was added and cells were cultured for an additional $4 \mathrm{~h}$. Subsequently, the medium was removed and $150 \mu \mathrm{l}$ DMSO was added to each well. After 5 min of shaking at $\mathrm{RT}$, the absorbance was measured at $570 \mathrm{~nm}$ by a microplate reader (BioTek Instruments, Winooski, VT, USA). Assays were performed in sextuplicate and repeated four times independently. Based on the results, $10^{-4} \mathrm{mmol} / \mathrm{L}$ of Ang II was determined to be the optimal treatment concentration for subsequent studies.

MTT assay was also used to examine the inhibitory effects of QYGS on proliferation in Ang II-treated HMCs. The cells were cultured as described above, and then were pre-treated with VS or QYGS in groups as follows: the vehicle group (with medium containing 5\% drug-free rat serum), the Ang II model group (with medium containing 5\% drug-free rat serum), the $5 \%$ VS group (positive control, with medium containing $5 \% \mathrm{VS}$ ), the $1.25 \%$ QYGS group (with medium containing 1.25\% QYGS and $3.75 \%$ drug-free rat serum), the $2.5 \%$ QYGS group (with medium containing 2.5\% QYGS and 2.5\% drug-free rat serum), and the $5 \%$ QYGS group (with medium containing 5\% QYGS). After one hour, all groups except for the vehicle group were incubated with $10^{-4} \mathrm{mmol} / \mathrm{L}$ of Ang II for $24 \mathrm{~h}$. Then, MTT assay was performed to detect cell proliferation in each group. Assays were performed in sextuplicate and repeated four times independently.

\section{Real time-polymerase chain reaction analysis}

HMCs were cultured in 6-well plates at a density of $2 \times 10^{5}$ cells per well. Then, the cells were grouped and 
pre-treated with VS or QYGS as described in the above section. One hour later, all groups except for the vehicle group were treated with $10^{-4} \mathrm{mmol} / \mathrm{L}$ of Ang II for $1 \mathrm{~h}$. Afterwards, total RNA was extracted from cells using TRIzol Plus reagent (Biouniquer) according to the manufacturer's instructions. RNA concentrations were determined by a microplate reader (BioTek Instruments). Total cDNA was synthesized with a cDNA synthesis kit (Biouniquer). RT-PCR was performed using an ABI 7700 Prism Sequence Detection System and TaqMan primer probes (Applied Biosystems, Foster City, CA, USA). The primer sequences are presented in Table 3. The total reaction volume was $20 \mu \mathrm{l}: 5 \mu \mathrm{l} \mathrm{cDNA}$, $10 \mu \mathrm{l} 2 \times$ UltraSYBR Mixture (with ROX), $0.5 \mu \mathrm{l}$ of each primer, and $4 \mu \mathrm{l}$ RNase-free water. Cycle parameters were as follows: activation at $95^{\circ} \mathrm{C}$ for $10 \mathrm{~min}, 40$ cycles of denaturation at $95^{\circ} \mathrm{C}$ for $15 \mathrm{~s}$, and then annealing and extension at $60^{\circ} \mathrm{C}$ for $60 \mathrm{~s}$. Calculations of the expression levels were carried out using the absolute standard curve method [18]. Glyceraldehyde-3-phosphate dehydrogenase (GAPDH) was used as an internal control. All experiments were conducted three times independently.

\section{Western blot analysis}

HMCs were cultured, grouped, and treated as described in the "Real time-polymerase chain reaction analysis" section. HMCs of all groups were then harvested by centrifugation at $1500 \mathrm{rpm}$ for $5 \mathrm{~min}$. The harvested cells were washed three times with phosphate-buffered saline (PBS, pH 7.2 - 7.4) and lysed in RIPA buffer by shaking at $4^{\circ} \mathrm{C}$ for $30 \mathrm{~min}$. The homogenates were centrifuged at 14,000 rpm for $10 \mathrm{~min}$ and the supernatant was used for western blot analysis. Protein concentrations were determined using the Bradford method according to the manufacturer's instructions (Beyotime Technology, Jiangsu, China). Supernatants were mixed with sodium dodecyl sulfate-polyacrylamidegel electrophoresis (SDSPAGE) sample buffer and boiled for $10 \mathrm{~min}$, and were then separated on 10\% SDS-PAGE gel. Subsequently,

Table 3 Primers used in RT-PCR

\begin{tabular}{ll}
\hline Target gene & Sequence of forward and reverse primers \\
\hline NOX4 & 5'-TAGATACCCACCCTCCCG-3' \\
& 5'-TGACTGGCTTATTGCTCC-3' \\
p22 phox & 5'- GCGGCATCTACCTACTGGC-3' \\
& 5'- CTCCTCGCTGGGCTTCTT-3' \\
Rac1 & 5'-GAAGCAGCAGAAATCACAG-3' \\
& 5'-CCAATACTCCAGAGGCAAG-3' \\
GTP-Rac1 & 5'-TTAGGGATGATAAAGACACG-3' \\
& 5'-GGACAGGACCAAGAACGAG-3' \\
GAPDH & 5'- GGATTTGGTCGTATTGGG-3' \\
& 5'- GGAAGATGGTGATGGGATT -3' \\
\hline
\end{tabular}

samples were transferred onto polyvinylidene difluoride (PVDF) membranes (Bio-Rad, Hercules, CA, USA). Membranes were blocked for $1 \mathrm{~h}$ in $5 \%(\mathrm{w} / \mathrm{v})$ non-fat milk and then incubated with a primary antibody at RT for $2 \mathrm{~h}$. The following primary antibodies were used: NOX4, p22 $2^{\text {phox }}$, Rac1, tumor necrosis factor- $\alpha$ (TNF- $\alpha$ ), NF- $k B$ p65, and interleukin 6 (IL-6) (Santa Cruz Biotechnology, Santa Cruz, CA, USA); activated Ras-related C3 botulinum toxin substrate 1 (GTP-Rac1) (NewEast Bioscience, Malvern, PA, USA); and $\beta$-actin (Sino Biological Inc., Beijing, China). Following incubation, the membranes were washed three times in TBST buffer, and then incubated with horseradish peroxidase (HRP)-conjugated rabbit IgG (diluted 1:5000) for $1 \mathrm{~h}$ at RT. After three washes with TBST, proteins were detected using an enhanced chemiluminescence (ECL) detection kit (Beyotime). The immunoblotted bands were quantified by Gel-Pro Analyzer software (version 4.0, Media Cybernetics, Rockville, MD, USA), and the protein of interest was normalized to $\beta$-actin. All experiments were performed three times independently.

\section{Reactive oxygen species generation}

HMCs were cultured, grouped, and treated as described in the "Real time-polymerase chain reaction analysis" section. Then, $2^{\prime}, 7^{\prime}$-dichlorodihydrofluorescein diacetate (DCHF-DA) (Beyotime) was added to each culture at a final concentration of $10 \mu \mathrm{mol} / \mathrm{L}$ and incubated for $20 \mathrm{~min}$ at $37^{\circ} \mathrm{C}$. The cells were washed with $\mathrm{PBS}$ three times and cellular fluorescence was assayed using cell flow cytometry (BD Bioscience, Bedford, MA, USA). Assays were repeated three times independently.

\section{Small interfering RNA against NOX4}

Twenty-four hours prior to transfection, $2 \times 10^{5} \mathrm{HMCs}$ were seeded into 6-well plates and cultured in antibiotic-free medium to obtain $50-80 \%$ confluence. Then, transfection of $125 \mathrm{pmol}$ small interfering RNA (siRNA) against NOX4 was performed using Lipofectamine 2000 (Life Technologies) according to the manufacturer's instructions. The transfected HMCs were grouped and treated the same way as described previously. A nonspecific scrambled siRNA was used as a negative control (the RNAcon group). Cells from all groups were subjected to RT-PCR analysis, Western blot, and ROS detection assay.

\section{Statistical analysis}

All statistical analysis was performed using SPSS 17.0 software (SPSS Inc., Chicago, IL, USA). Data are presented as means \pm standard error of the mean (SEM). Differences between experimental groups are assessed for statistical significance using one-way analysis of variance (ANOVA), followed by least significant difference 
(LSD) or Games-Howell post-hoc multiple comparison tests for equal or unequal variances, respectively. A $P$ value less than 0.05 was considered statistically significant (two-tailed).

\section{Results}

Effects of Ang II on proliferation of HMGs

The effects of Ang II on proliferation of HMCs are shown in Figure 1 . We found that only $10^{-4} \mathrm{mmol} / \mathrm{L}$ of Ang II could significantly promote the proliferation of HMCs when the cells were treated with various doses of Ang II $\left(10^{-3}\right.$ to $10^{-7} \mathrm{mmol} / \mathrm{L}$ in a 10 -fold concentration gradient) for $24 \mathrm{~h}$. Therefore, $10^{-4} \mathrm{mmol} / \mathrm{L}$ of Ang II was used in subsequent experiments.

\section{Inhibitory effects of QYGS on Ang Il-induced HMC proliferation}

Figure 2 presents the inhibitory effects of QYGS on Ang II-induced HMC proliferation. As shown in Figure 2A and $\mathrm{B}$, up to $5 \%$ concentrations of either VS or QYGS alone did not influence the proliferation of HMCs. When HMCs were pre-incubated with various doses of VS or QYGS $(1.25 \%, 2.5 \%$, and $5 \%)$ for $1 \mathrm{~h}$ prior to treatment with $10^{-4} \mathrm{mmol} / \mathrm{L}$ of Ang II, cell proliferation was significantly decreased in a dose-dependent manner, thereby indicating that both VS and QYGS possess a potent inhibitory activity against Ang II-induced HMC proliferation (Figure 2C and D).

\section{Inhibitory effects of QYGS on Ang II-induced ROS production in HMCs}

Figure 3 shows the inhibitory effects of QYGS on Ang IIinduced ROS production in HMCs. Using the DCFH-DA method, we found a significant production of ROS when

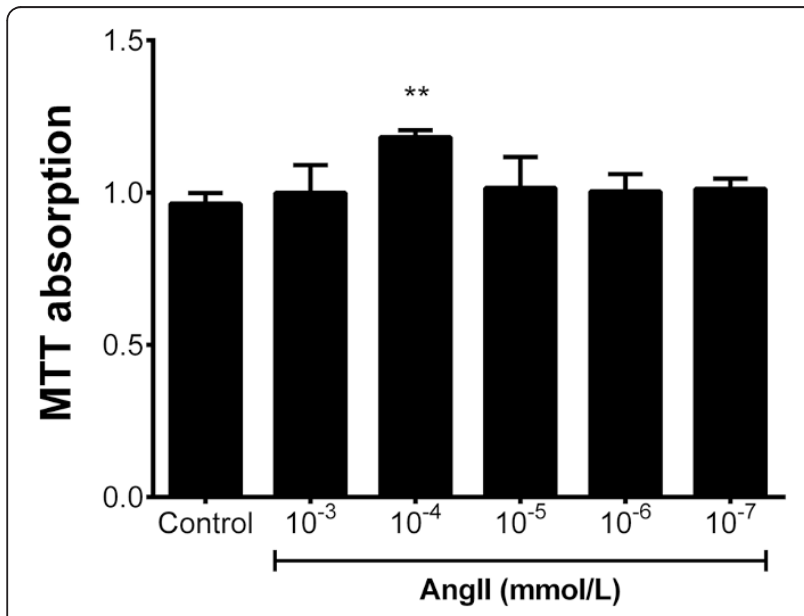

Figure 1 Effects of Ang II on HMC proliferation after 24 h-treatment. Cell proliferation was assessed using the MTT method. Data are presented as means \pm SEM $(n=4)$. ${ }^{* *} P<0.01$ compared with the control group.
HMCs were treated with $10^{-4} \mathrm{mmol} / \mathrm{L}$ of Ang II for $24 \mathrm{~h}$ (Figure 3). However, ROS levels were considerably decreased after pre-incubation with $5 \%$ VS or with $1.25 \%$, $2.5 \%$, or $5 \%$ QYGS for $1 \mathrm{~h}$ prior to Ang II treatment. In addition, a dose-dependent effect was observed for QYGS pre-incubation. Collectively, these findings demonstrate that QYGS is able to suppress Ang II-induced ROS generation in HMCs.

Effects of QYGS on mRNA and protein expression of NOX4, p22 ${ }^{\text {phox }}$, and Rac1/GTP-Rac1 in Ang Il-treated HMCs

As previously reported, Ang II could induce activation of NOX4 and Rac1 in mesangial cells. NOX4 forms a heterodimer with $\mathrm{p} 22^{\text {phox }}$ subunits to facilitate ROS generation, while Rac1 uses Nox4-derived ROS as signal transducers to stimulate downstream signaling cascades [19]. In combining this information with the results shown in Figure 3, we assumed that the inhibitory effects of QYGS on Ang II-induced ROS production in HMCs may be mediated by a NOX4-dependent pathway.

In the current study, we found that treatment with $10^{-4} \mathrm{mmol} / \mathrm{L}$ of Ang II could induce a significant increase in mRNA and protein levels of NOX4, p22 phox, and activated GTP-Rac1 (Figure 4A and B). Nevertheless, these effects could be counteracted by $5 \%$ VS or by QYGS in a dose-dependent manner (from $1.25 \%$ to $5 \%)$. For Rac1, however, no notable difference was observed in its mRNA and protein expression among all the groups. Taken together, these findings provide supportive evidence that QYGS is able to suppress the activation of the NOX4-dependent pathway in Ang II-treated HMCs, which may be responsible for attenuating Ang IIinduced ROS production.

Effects of QYGS on expression of TNF- $a$, NF-KB p65, and IL-6 in Ang II-treated HMCs

In taking into consideration that ROS-induced oxidative stress plays an essential role in activating inflammatory pathways in pro-inflammatory responses [20], we investigated the effects of QYGS on the expression of inflammatory markers, including TNF- $\alpha$, NF- $\mathrm{kB}$ p65, and IL-6. As shown in Figure 5, treatment with $10^{-4} \mathrm{mmol} / \mathrm{L}$ of Ang II induced a significant increase in the protein expression of these inflammatory markers. After incubation with $5 \%$ VS or with $1.25 \%, 2.5 \%$, or $5 \%$ QYGS for $1 \mathrm{~h}$ prior to Ang II treatment, the effects of Ang II on the expression of the three marker proteins were significantly counteracted. These results indicate that QYGS is able to prevent Ang II-induced pro-inflammatory responses in HMCs.

\section{Silencing RNA against NOX4 in Ang II-treated HMCs}

For further analysis, we employed siRNA to silence NOX4 expression in HMCs. After transfection with NOX4 

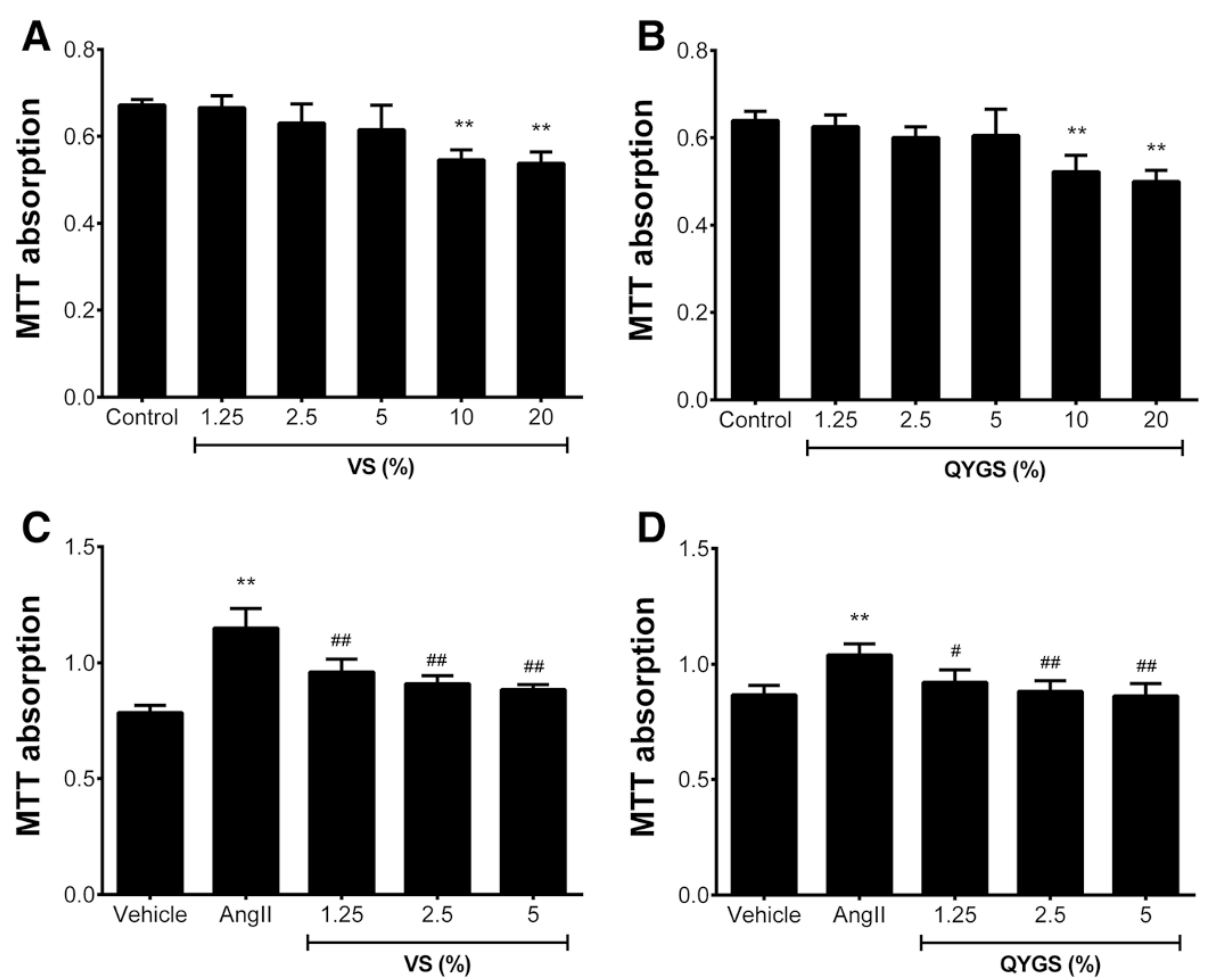

Figure 2 Effects of QYGS on Ang II-induced HMC proliferation assessed by MTT assay. (A) Effect of VS (0, 1.25\%, 2.5\%, 5\%, 10\%, and 20\%) on cell proliferation after 24 h-incubation. (B) Effect of QYGS $(0,1.25 \%, 2.5 \%, 5 \%, 10 \%$, and 20\%) on HMC proliferation following incubation for 24 h. (C) and (D) Inhibitory effects of varying doses of VS or QYGS (1.25\%, 2.5\%, and 5\%) on cell proliferation in Ang II-treated HMCs. Data are presented as means \pm SEM $(n=4) .{ }^{*} P<0.01$ as compared with the control group or the vehicle group. ${ }^{\#} P<0.05,{ }^{\# \#} P<0.01$ as compared with the Ang II model group.

siRNA for $24 \mathrm{~h}$, RT-PCR showed that the mRNA expression of NOX4, p22 $2^{\text {phox }}$, and GTP-Rac1 in HMCs had decreased by $72.7 \%, 69.3 \%$, and $61.8 \%$, respectively, which was statistically significant as compared with the RNAcon group (Figure 6A). These results demonstrate that the

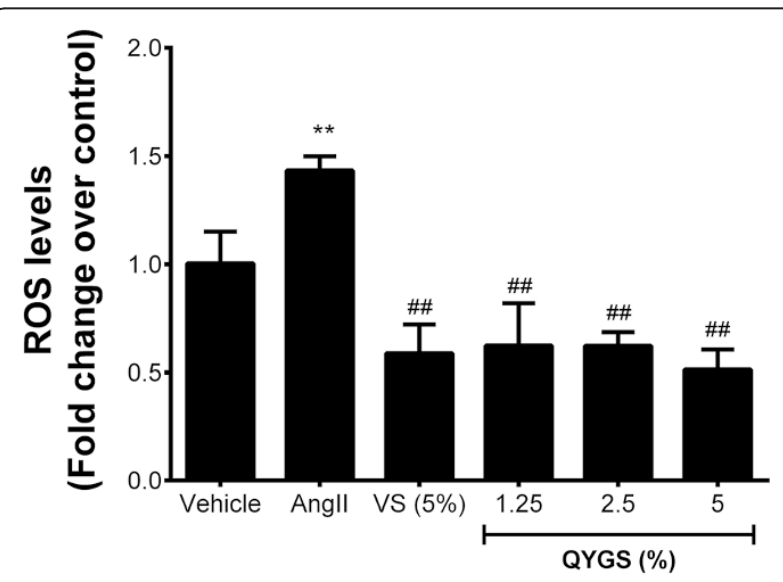

Figure 3 Effects of QYGS on Ang II-induced ROS production in HMCs assessed by DCFH-DA assay. Data are expressed as means \pm SEM $(n=3) .{ }^{*} P<0.01$ as compared with the vehicle group. ${ }^{\# \#} P<0.01$ as compared with the Ang II model group. transfection of NOX4 siRNA was successful with a silencing efficiency of over $70 \%$. After cells transfected with NOX4 siRNA were treated with $10^{-4} \mathrm{mmol} / \mathrm{L}$ of Ang II for $24 \mathrm{~h}$, we found that mRNA expression levels of $\mathrm{p} 22^{\text {phox }}$ and GTP-Rac1 were still significantly increased as compared with the vehicle group, indicating that the NADPHoxidase activity was not completely inhibited upon siRNA knockdown of NOX4 and that other NADPH-oxidases may be activated by Ang II, thereby leading to upregulation of $\mathrm{p} 22^{\text {phox }}$ and GTP-Rac1 expression. When cells were pre-incubated with $5 \%$ VS or with $1.25 \%, 2.5 \%$, or 5\% QYGS for $1 \mathrm{~h}$ followed by Ang II stimulation, the expression levels of $\mathrm{p} 22^{\text {phox }}$ and GTP-Rac1 were significantly decreased; and a dose-dependent effect was noted for QYGS pre-incubation. For Rac1, however, no significant difference was observed in mRNA levels among the groups overall. Similar results showing decreased protein levels of $\mathrm{p} 22^{\text {phox }}$ and GTP-Rac1 in QYGS-pretreated HMCs were also obtained by Western blot analysis (Figure 6B).

Furthermore, we examined ROS production in siRNAtransfected HMCs, the results of which are shown in Figure 7. After transfection with NOX4 siRNA for $24 \mathrm{~h}$, DCFH-DA assay showed that the ROS production in 


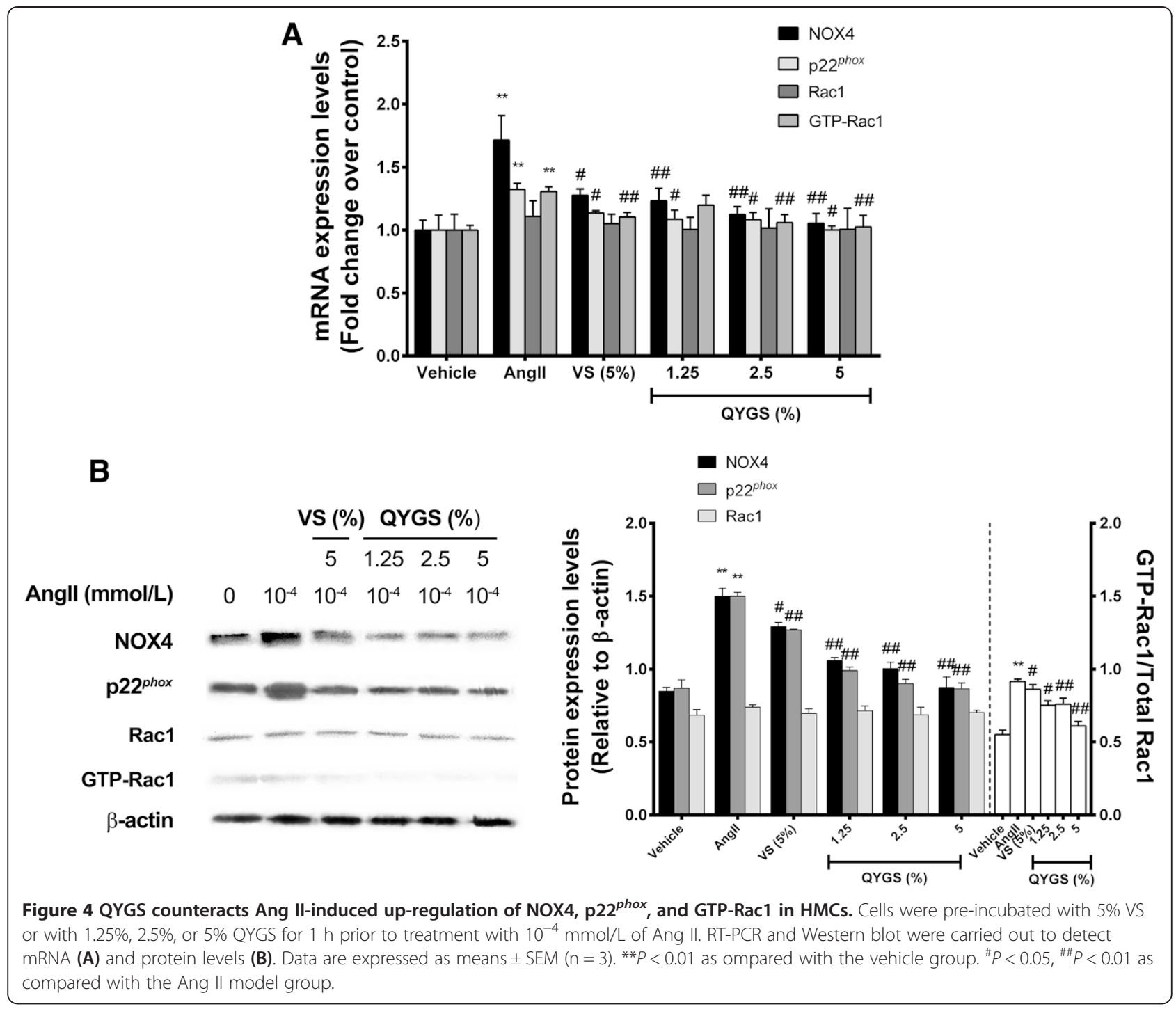

HMCs was significantly reduced when compared with the RNAcon group. However, treatment with $10^{-4} \mathrm{mmol} / \mathrm{L}$ of Ang II was able to induce a considerable increase in ROS production in siRNA-transfected cells. When cells were pre-treated with $5 \%$ VS or various doses of QYGS, this inductive effect of Ang II could be significantly inhibited, further demonstrating the ability of QYGS to inhibit Ang II-induced ROS generation in HMCs.

In order to further verify the effects of QYGS on suppressing Ang II-induced pro-inflammatory responses, we

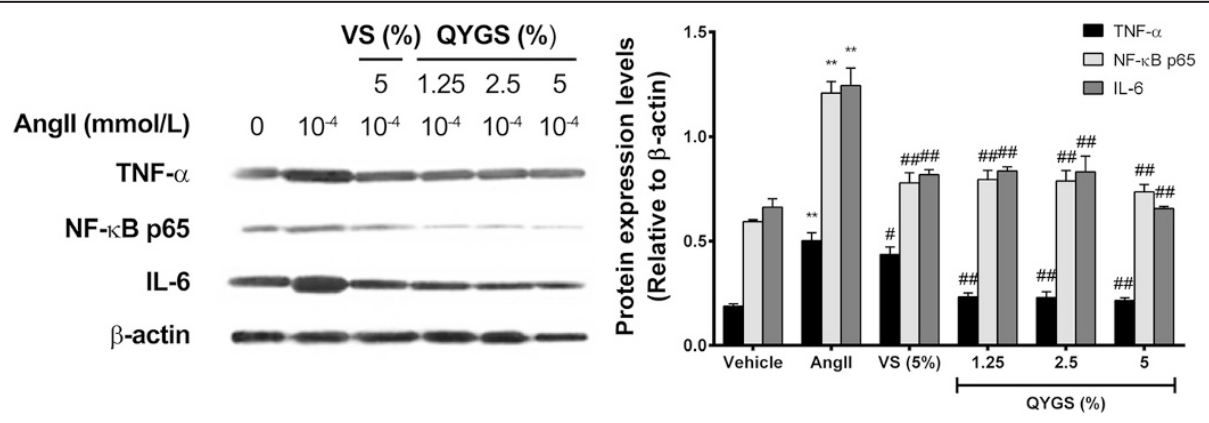

Figure 5 Effects of QYGS on protein expression of TNF-a, NF-KB P65, and IL-6 in Ang II-treated HMCs as assessed by Western blot. Data are presented as means \pm SEM $(n=3)$. ${ }^{* *} P<0.01$ as compared with the vehicle group. ${ }^{\#} P<0.05$, ${ }^{\# \#} P<0.01$ as compared with the Ang $\|$ model group. 


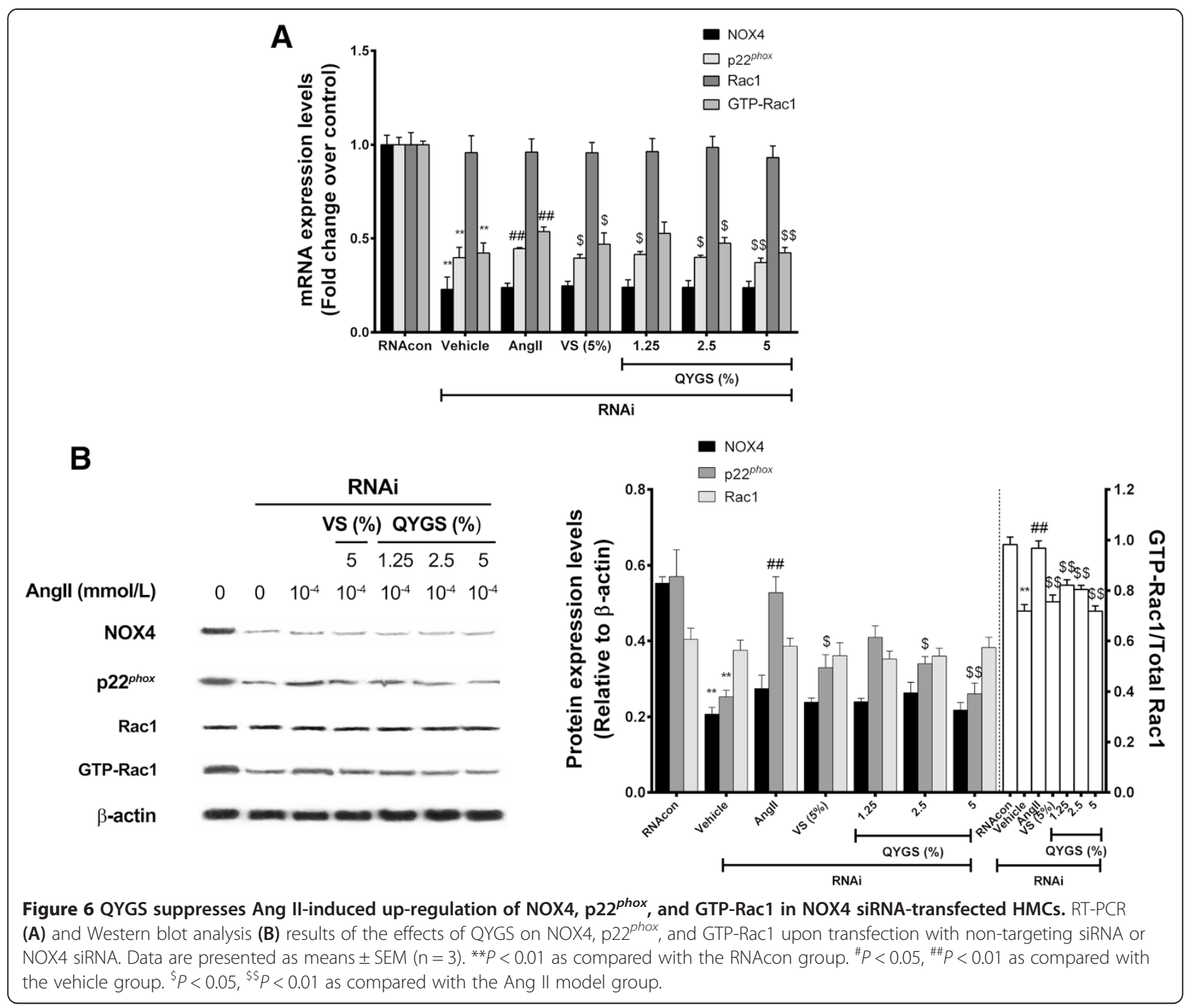

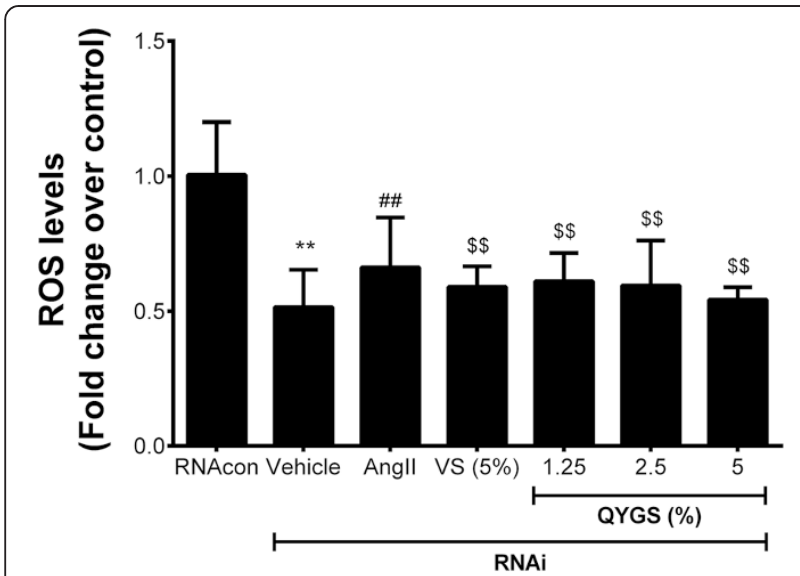

Figure 7 Effects of QYGS on Ang II-induced ROS production in NOX4 siRNA-transfected HMCs as assessed by DCFH-DA assay. Data are expressed as means \pm SEM $(n=3) .{ }^{* *} P<0.01$ as compared with the RNAcon group. ${ }^{\#} P<0.01$ as compared with the vehicle group. ${ }^{\$} P<0.01$ as compared with the Ang II model group. detected the protein levels of TNF- $\alpha$, NF-кB p65, and IL-6 in HMCs after siRNA transfection. As shown in Figure 8, siRNA knockdown of NOX4 led to a considerable decrease in expression of the three inflammatory marker proteins as compared to the RNAcon group. When cells were incubated with Ang II, incubation caused a notable increase in the protein levels of all inflammatory markers. However, this effect was counteracted by pre-treatment with $5 \%$ VS or various doses of QYGS.

\section{Discussion}

In the current study, we report for the first time that QYGS has inhibitory effects on Ang II-induced proliferation in HMCs. Furthermore, we found that QYGS is able to attenuate Ang II-induced ROS generation and proinflammatory responses in cells. These effects may be mediated by an NOX4-dependent pathway. 

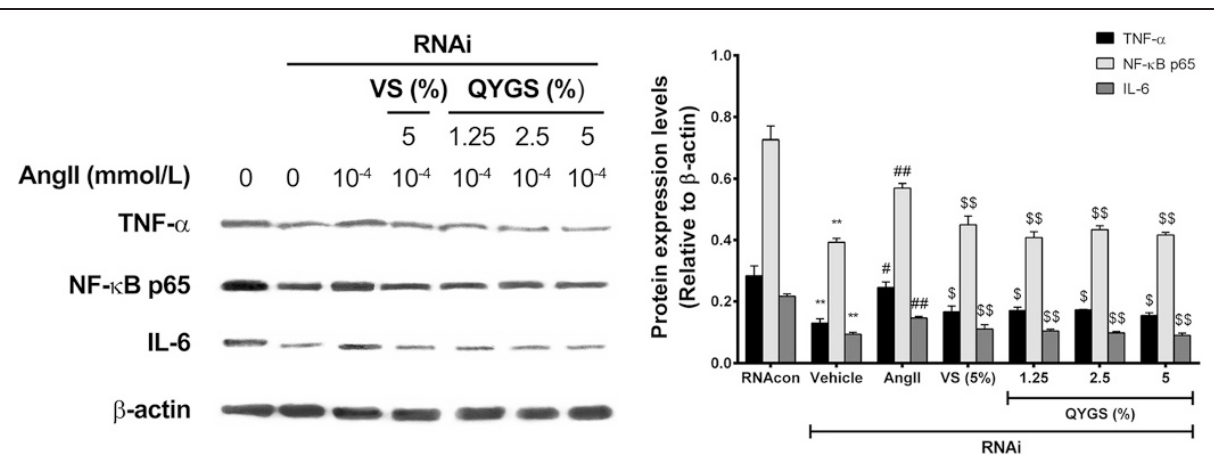

Figure 8 Effects of QYGS on Ang II-mediated up-regulation TNF-a, NF-KB P65, and IL-6 in NOX4 siRNA-transfected HMCs as assessed by Western blot analysis. Data are presented as means \pm SEM $(n=3)$. ${ }^{* *} P<0.01$ as compared with the RNAcon group. ${ }^{\#} P<0.05,{ }^{\# \#} P<0.01$ as compared with the vehicle group. ${ }^{\$} P<0.01$ as compared with the Ang II model group.

Renal vascular remodeling, hyperplasia of the glomerular mesangium, and renal fibrosis have been considered to be the main pathogenic factors behind renal damage in hypertension [21]. Hyperplasia of the glomerular mesangium is characterized by the proliferation of glomerular mesangial cells and excessive ECM accumulation [21,22]; while the key feature of renal fibrosis is the accumulation of myofibroblasts and ECM deposition [23]. Therefore, both the proliferation of glomerular mesangial cells and ECM deposition have been implicated as two key events in the progression of renal damage [24]. RAS, and particularly Ang II, is well known to be involved in a wide variety of cardiovascular pathologies including hypertension [2]. Researchers have demonstrated that Ang II is able to stimulate glomerular mesangial cell proliferation and ECM deposition, consequently causing glomerular sclerosis and additional renal damage [3]. It is therefore widely accepted that inhibition of Ang II-induced glomerular mesangial cell proliferation and ECM deposition should be an effective approach to preventing the development or progression of renal damage in hypertension [25,26]. In the current study, we found that pre-treatment with various doses of QYGS (from $1.25 \%$ to $5 \%$ ), like pretreatment with 5\% VS, could effectively inhibit Ang II-induced HMC proliferation. This effect may be due to the antioxidant and anti-inflammatory properties of the active components of QYYYG.

QYYYG contains six Chinese medical herbs, of which Polygoni Multiflori Radix is the major active component and has been indicated for the treatment of hypertension and hypertension-related renal diseases in Chinese Traditional Medicine for a number of decades. In recent years, numerous studies have proven that 2,3,5,4'-tetrahydroxystilbene-2-O- $\beta$-D-glucopyranoside (THSG) is the main active substance in Polygoni Multiflori Radix, which produces potent activity against the oxidative stress that follows acute inflammation [27-29]. In our study, although QYGS was used instead of QYYYG as intervention in HMCs, HPLC analysis showed that there was a high content of THSG in QYGS (data not shown). Furthermore, researchers have reported that some active substances extracted from Corni Fructus, such as 7-Ogalloyl-D-sedoheptulose and loganin, have potent protective effects against diabetes- or glycerol-induced renal injury through elimination of ROS and inhibition of inflammation [30,31]. Therefore, we assume that the antioxidant and anti-inflammatory activity of these compounds may be involved in the inhibitory effect of QYGS on Ang II-induced HMC proliferation.

Recently, many studies have confirmed that oxidative stress and inflammation are important hallmarks of renal damage in hypertension [32,33]. As the key component of RAS, Ang II induces oxidative stress and inflammation by activating the Ang II type 1 (AT1) receptor [34]. Under pathological conditions, inflammatory changes induced by Ang II can lead to proliferation of mesangial cells and loss of the mesangial matrix, followed by excessive production of ECM and mesangial expansion [2]. Growing evidence suggests that these effects of Ang II are mainly mediated via activation of NOXs [7]. Researchers have demonstrated that NOXs are widely expressed in renal tissue [35]. Currently, at least four NOXs (NOX1, NOX2, NOX4, and NOX5) have been identified, of which NOX4 shows the highest expression level [36]. Recent studies have indicated that NOX4 is associated with p $22^{\text {phox }}$ and serves as an important source of ROS; while Rac1 uses Nox4-derived ROS to elicit downstream signaling pathways [36,37]. Gorin et al. have provided definitive evidence that Ang II is able to induce activation of NOX4 and Rac1 in mesangial cells [19]. In line with their findings, we also found that incubation with $10^{-4} \mathrm{mmol} / \mathrm{L}$ of Ang II could not only induce a significant increase in ROS production in HMCs, but could also considerably enhance the mRNA and protein levels of NOX4, p22 $2^{\text {phox }}$, and activated GTP-Rac1 as compared to the vehicle group. When 
cells were pre-treated with QYGS, we found that QYGS was able to decrease ROS production and downregulate mRNA and protein expression of NOX4, p22 $2^{\text {phox }}$, and GTP-Rac1 in Ang II-treated HMCs. These results suggest that these effects of QYGS may be mediated through a NOX4-dependent pathway. Nevertheless, when NOX4 was silenced by siRNA in HMCs, Ang II was able to facilitate ROS production and expression of the aforementioned molecules to at least some extent; but these effects were still counteracted by intervention with QYGS. According to previous literature, Ang II is also functionally associated with NOX1, NOX2, and NOX5 [36]. Therefore, these findings imply that other NOXs might also be involved in the antioxidant potency of QYGS. However, extensive studies are required to confirm this.

Numerous studies have revealed that ROS production upon Ang II-induced NOX activation promotes downstream inflammatory responses, which are critical for the development and progression of renal damage in hypertension $[38,39]$. The signaling pathways involved in this process include NF- $\mathrm{KB}$, STAT, and MAPK pathways, which can be activated by ROS to regulate the gene expression of numerous cytokines, as well as adhesion and chemoattractant molecules [40]. In this study, we found that QYGS is able to inhibit the Ang II-induced protein expression of TNF- $\alpha$, NF- $k B$ p65, and IL-6 in HMCs. This finding demonstrates the inhibitory effect of QYGS on Ang II-induced pro-inflammatory responses, which is assumed to be related to the negative impact of QYGS on Ang II/NOX pathway. However, a thorough understanding of how the active ingredients in QYGS, such as THSG, exert their signaling functions still awaits further research.

One possible limitation to this study should be noted. According to Cao et al., rat and human exhibit different expression levels and patterns for metabolizing enzymes in the intestine [41]. Hence, it is reasonable to assume that the metabolism of QYYYG in rat may be different with that in human, and that the use of QYGS might introduce a bias into the results of this study. Therefore, further studies using QYYYGcontaining human serum are required to validate the present findings.

\section{Conclusions}

In summary, the current study shows that QYGS is able to inhibit cell proliferation, ROS production, and inflammation in Ang-II-treated HMCs. Our data suggest that an NOX4-dependent pathway plays an important role in regulating the inhibitory effect of QYGS. These findings provide new insights into the molecular mechanisms of QYYYG in the treatment of renal damage in hypertension.

\section{Abbreviations}

Ang II: Angiotensin II; ARBs: Angiotensin receptor blockers; DCHF-DA: 2',7'-dichlorodihydrofluorescein diacetate; DMSO: Dimethyl sulfoxide; ECM: Extracellular matrix; GTP-Rac1: Activated Ras-related C3 botulinum toxin substrate 1; HMCs: Human mesangial cells; IL-6: Interleukin 6; MTT: 3-(4,5-dimethyl-2-thiazolyl)-2,5-diphenyl tetrazolium bromide; NF-KB p65: Nuclear factor-kB p65; NOX: Nicotinamide adenine dinucleotide phosphate (NAPDH)-oxidase; QYGS: QYYYG-containing serum; QYYYG: Qian Yang Yu Yin Granule; RAS: The renin-angiotensin system; ROS: Reactive oxygen species; siRNA: Small interfering RNA; TNF-a: Tumor necrosis factor-a; VS: Valsartan-containing serum.

\section{Competing interests}

The authors declare that they have no competing interests.

\section{Authors' contributions}

KD and YW carried out all the experiments and drafted the manuscript. WJ participated in the design of the study. YZ and HY participated in the design of the study and performed the statistical analysis. ZF conceived of the study, and participated in its design and coordination and helped to draft the manuscript. All authors read and approved the final manuscript.

\section{Acknowledgments}

This study was supported by the Research Fund for Doctoral Program of Higher Education of China (Grant No. 20123237110005) and the National Nature Science Foundation of China (Grant No. 81273713). This study was a project funded by the Priority Academic Program Development of Jiangsu Higher Education Institutions.

\section{Author details}

'First College of Clinical Medicine, Nanjing University of Traditional Chinese Medicine, Nanjing 210046, China. ${ }^{2}$ Nanjing Hospital of Traditional Chinese Medicine, Nanjing 210001, China. ${ }^{3}$ Jiangsu Province Hospital of Traditional Chinese Medicine, No.155 Hanzhong Road, Nanjing 210029, China. ${ }^{4}$ School of Life Science and Technology, China Pharmaceutical University, Nanjing 210009, China. ${ }^{5}$ College of Pharmacy, Nanjing University of Traditional Chinese Medicine, Nanjing 210029, China.

Received: 6 October 2014 Accepted: 16 March 2015

Published online: 25 March 2015

\section{References}

1. Bakris GL. Hypertension in 2011: new insights-from risk factors to treatment implications. Nat Rev Cardiol. 2012;9(2):75-7.

2. Santos PC, Krieger JE, Pereira AC. Renin-angiotensin system, hypertension, and chronic kidney disease: pharmacogenetic implications. J Pharmacol Sci. 2012;120(2):77-88.

3. Kobori H, Mori H, Masaki T, Nishiyama A. Angiotensin II blockade and renal protection. Curr Pharm Des. 2013;19(17):3033-42

4. Gorin Y, Ricono JM, Wagner B, Kim NH, Bhandari B, Choudhury GG, et al. Angiotensin I-induced ERK1/ERK2 activation and protein synthesis are redox-dependent in glomerular mesangial cells. Biochem J. 2004;381(Pt 1):231-9.

5. Block K, Eid A, Griendling KK, Lee DY, Wittrant Y, Gorin Y. Nox4 NAD(P)H oxidase mediates Src-dependent tyrosine phosphorylation of PDK-1 in response to angiotensin $\mathrm{I}$ : role in mesangial cell hypertrophy and fibronectin expression. J Biol Chem. 2008;283(35):24061-76.

6. Gabor A, Leenen FH. Central neuromodulatory pathways regulating sympathetic activity in hypertension. J Appl Physiol (1985). 2012;113(8):1294-303.

7. Gill PS, Wilcox CS. NADPH oxidases in the kidney. Antioxid Redox Signal. 2006:8(9-10):1597-607.

8. Kim S, Iwao H. Molecular and cellular mechanisms of angiotensin II-mediated cardiovascular and renal diseases. Pharmacol Rev. 2000;52(1):11-34.

9. Bedard K, Krause KH. The NOX family of ROS-generating NADPH oxidases: physiology and pathophysiology. Physiol Rev. 2007;87(1):245-313.

10. Covic A, Gusbeth-Tatomir P. The role of the renin-angiotensin-aldosterone system in renal artery stenosis, renovascular hypertension, and ischemic nephropathy: diagnostic implications. Prog Cardiovasc Dis. 2009;52(3):204-8.

11. Mancia G, Fagard R, Narkiewicz K, Redon J, Zanchetti A, Bohm M, et al. 2013 ESH/ESC Guidelines for the management of arterial hypertension: the Task Force for the management of arterial hypertension of the European Society 
of Hypertension (ESH) and of the European Society of Cardiology (ESC). J Hypertens. 2013;31(7):1281-357.

12. Zhang J, Noble NA, Border WA, Huang Y. Infusion of angiotensin-(1-7) reduces glomerulosclerosis through counteracting angiotensin II in experimental glomerulonephritis. Am J Physiol Renal Physiol. 2010;298(3):F579-88.

13. Shao J, Nangaku M, Inagi R, Kato H, Miyata T, Matsusaka T, et al. Receptorindependent intracellular radical scavenging activity of an angiotensin II receptor blocker. J Hypertens. 2007;25(8):1643-9.

14. Fliser D, Buchholz K, Haller H, Olmesartan EUTo, Pravastatin in I, Atherosclerosis I. Antiinflammatory effects of angiotensin II subtype 1 receptor blockade in hypertensive patients with microinflammation. Circulation. 2004;110(9):1103-7.

15. Jalili J, Askeroglu U, Alleyne B, Guyuron B. Herbal products that may contribute to hypertension. Plast Reconstr Surg. 2013;131(1):168-73.

16. Yu J, Xie J, Mao XJ, Wei H, Zhao SL, Ma YG, et al. Comparison of laxative and antioxidant activities of raw, processed and fermented Polygoni Multiflori radix. Chin J Nat Med. 2012;10(1):63-7.

17. Huang $C H$, Horng LY, Chen CF, Wu RT. Chinese herb Radix Polygoni Multiflori as a therapeutic drug for liver cirrhosis in mice. J Ethnopharmacol. 2007;114(2):199-206.

18. Larionov A, Krause A, Miller W. A standard curve based method for relative real time PCR data processing. BMC Bioinformatics. 2005;6:62.

19. Gorin Y, Ricono JM, Kim NH, Bhandari B, Choudhury GG, Abboud HE. Nox4 mediates angiotensin II-induced activation of Akt/protein kinase B in mesangial cells. Am J Physiol Renal Physiol. 2003;285(2):F219-29.

20. Sachse A, Wolf G. Angiotensin II-induced reactive oxygen species and the kidney. J Am Soc Nephrol. 2007;18(9):2439-46.

21. Li JM, Shah AM. ROS generation by nonphagocytic NADPH oxidase: potential relevance in diabetic nephropathy. J Am Soc Nephrol. 2003;14(8 Suppl 3):S221-6.

22. Dubey RK, Jackson EK, Rupprecht HD, Sterzel RB. Factors controlling growth and matrix production in vascular smooth muscle and glomerular mesangial cells. Curr Opin Nephrol Hypertens. 1997;6(1):88-105.

23. Kieseier BC, Dalakas MC, Hartung HP. Immune mechanisms in chronic inflammatory demyelinating neuropathy. Neurology. 2002;59(12 Suppl 6):S7-12.

24. Migliorini A, Ebid R, Scherbaum CR, Anders HJ. The danger control concept in kidney disease: mesangial cells. J Nephrol. 2013;26(3):437-49.

25. Gomez-Guerrero C, Hernandez-Vargas P, Lopez-Franco O, Ortiz-Munoz G, Egido J. Mesangial cells and glomerular inflammation: from the pathogenesis to novel therapeutic approaches. Curr Drug Targets Inflamm Allergy. 2005;4(3):341-51.

26. Okada H, Kalluri R. Cellular and molecular pathways that lead to progression and regression of renal fibrogenesis. Curr Mol Med. 2005;5(5):467-74.

27. Wang X, Zhao L, Han T, Chen S, Wang J. Protective effects of 2,3,5,4'tetrahydroxystilbene-2-O-beta-d-glucoside, an active component of Polygonum multiflorum Thunb, on experimental colitis in mice. Eur J Pharmacol. 2008:578(2-3):339-48.

28. Zhang YZ, Shen JF, Xu JY, Xiao JH, Wang JL. Inhibitory effects of 2,3,5,4'tetrahydroxystilbene-2-O-beta-D-glucoside on experimental inflammation and cyclooxygenase 2 activity. J Asian Nat Prod Res. 2007;9(3-5):355-63.

29. Yarosh DB, Galvin JW, Nay SL, Pena AV, Canning MT, Brown DA. Antiinflammatory activity in skin by biomimetic of Evodia rutaecarpa extract from traditional Chinese medicine. J Dermatol Sci. 2006;42(1):13-21.

30. Park CH, Noh JS, Tanaka T, Yokozawa T. 7-O-galloyl-D-sedoheptulose ameliorates renal damage triggered by reactive oxygen species-sensitive pathway of inflammation and apoptosis. J Pharm Pharmacol. 2012;64(12):1730-40.

31. Yamabe N, Noh JS, Park CH, Kang KS, Shibahara N, Tanaka T, et al. Evaluation of loganin, iridoid glycoside from Corni Fructus, on hepatic and renal glucolipotoxicity and inflammation in type 2 diabetic $\mathrm{db} / \mathrm{db}$ mice. Eur J Pharmacol. 2010;648(1-3):179-87.

32. Sung CC, Hsu YC, Chen CC, Lin YF, Wu CC. Oxidative stress and nucleic acid oxidation in patients with chronic kidney disease. Oxid Med Cell Longev. 2013;2013:301982.

33. Sedeek M, Nasrallah R, Touyz RM, Hebert RL. NADPH oxidases, reactive oxygen species, and the kidney: friend and foe. J Am Soc Nephrol. 2013;24(10):1512-8.

34. Welch WJ. Angiotensin II-dependent superoxide: effects on hypertension and vascular dysfunction. Hypertension. 2008;52(1):51-6.

35. Ponnuchamy B, Khalil RA. Cellular mediators of renal vascular dysfunction in hypertension. Am J Physiol Regul Integr Comp Physiol. 2009;296(4):R1001-18.
36. Nguyen Dinh Cat A, Montezano AC, Burger D, Touyz RM. Angiotensin II, NADPH oxidase, and redox signaling in the vasculature. Antioxid Redox Signal. 2013;19(10):1110-20.

37. Inoguchi T, Sonta T, Tsubouchi H, Etoh T, Kakimoto M, Sonoda N, et al. Protein kinase C-dependent increase in reactive oxygen species (ROS) production in vascular tissues of diabetes: role of vascular NAD(P)H oxidase. J Am Soc Nephrol. 2003;14(8 Suppl 3):S227-32.

38. Pacurari M, Kafoury R, Tchounwou PB, Ndebele K. The Renin-Angiotensinaldosterone system in vascular inflammation and remodeling. Int J Inflam. 2014;2014:689360.

39. Ferenbach $D$, Kluth $D C$, Hughes J. Inflammatory cells in renal injury and repair. Semin Nephrol. 2007;27(3):250-9.

40. Lan HY. Transforming growth factor-beta/Smad signalling in diabetic nephropathy. Clin Exp Pharmacol Physiol. 2012;39(8):731-8.

41. Cao X, Gibbs ST, Fang L, Miller HA, Landowski CP, Shin HC, et al. Why is it challenging to predict intestinal drug absorption and oral bioavailability in human using rat model. Pharm Res. 2006;23(8):1675-86,

\section{Submit your next manuscript to BioMed Central and take full advantage of:}

- Convenient online submission

- Thorough peer review

- No space constraints or color figure charges

- Immediate publication on acceptance

- Inclusion in PubMed, CAS, Scopus and Google Scholar

- Research which is freely available for redistribution 